\title{
INTERMEDIATE TEMPERATURE CREEP DEFORMATION IN CMSX-3
}

\author{
SINGLE CRYSTALS
}

\author{
Tresa M. Pollock and A.S. Argon \\ Massachusetts Institute of Technology, Cambridge, MA
}

\begin{abstract}
$\underline{\text { Abstract }}$
Creep deformation in $<001>$ oriented single crystals of CMSX-3 has been studied at temperatures around $850^{\circ} \mathrm{C}$ at a stress level of $552 \mathrm{MPa}$. Detailed observations of the evolution of the dislocation structure during creep have been made. TEM stereo pairs show creep deformation in the matrix phase, leaving the $\gamma^{\prime}$ precipitates undeformed. In previously undeformed crystals there is a brief incubation period during which dislocations spread from widely spaced sources, bowing out between the precipitates in the narrow matrix gaps. This Orowan bowing process is the major source of creep resistance. In the early stages of primary creep, over large regions dislocations all have the same Burgers vector in spite of the fact that there are eight equivalent slip systems. Later in the primary transient dislocations with other Burgers vectors interpenetrate from neighboring regions, gradually building up a three dimensional nodal network, which is characteristic of steady state creep. At $850^{\circ} \mathrm{C}$ static recovery processes are sluggish, indicating that dynamic recovery may be important at this temperature and at lower temperatures. Finite element analysis shows that the constraint of the non-deforming precipitates leads to the buildup of a negative pressure in the matrix channels normal to the applied stress. The negative pressure provides load support and reduces the effective stresses driving the steady state creep process in the matrix. The $\gamma^{\prime}$ precipitates are increasingly stressed as the creep process progresses, and under the conditons studied here, they are occasionally sheared in the later stages of steady state creep.
\end{abstract}




\section{Introduction}

Nickel base alloys have long been used in high temperature applications because of their exceptional creep resistance at high fractions of their melting temperature. Based on the bulk properties of the constituent $\gamma$ and $\gamma^{\prime}$ phases, continuum estimates of the creep strength greatly underestimate the actual properties of these two phase alloys. It is clear that while the presence of the high volume fraction of ordered precipitates must be altering the development of the creep substructure relative to what is observed in the bulk solid solution materials, that the actual scale of the $\gamma^{\prime}$ phase domains is also of key importance. For this reason a detailed study of the development of the dislocation structure during creep has been undertaken. The temperature range of $800^{\circ} \mathrm{C}$ to $900^{\circ} \mathrm{C}$ has been selected since in this range the $\gamma^{\prime}$ precipitates are stable with respect to rafting in the duration of a normal laboratory test. An understanding of the creep process at these intermediate temperatures should provide a meaningful starting point for dealing with the creep problem at higher temperatures where the precipitates are changing shape.

\section{Experimental Procedures}

CMSX-3 single crystals of $<001>$ orientation and nominal composition listed in (1) were used in this investigation. Samples were given a solution treatment of $1293^{\circ} \mathrm{C} / 2$ hours $+1298^{\circ} \mathrm{C} / 3$ hours. A two step aging treatment of $1080^{\circ} \mathrm{C} / 4$ hours $+870^{\circ} \mathrm{C} / 16$ hours produces 65-70 volume percent cuboidal gamma prime precipitates, $0.45 \mu \mathrm{m}$ in average diameter.

Prior to testing, samples were electropolished and thermocouples were welded to the sample surface for accurate temperature measurement. Creep experiments were run in a resistance heated vacuum furnace, with four LVDTs mounted on the loading rods for measurement of creep strains. The majority of the creep tests were at a temperature of $850^{\circ} \mathrm{C}\left(\mathrm{T} / \mathrm{T}_{m}=0.7\right)$ and a stress of $552 \mathrm{MPa}$. To assess the effects of static recovery on the mechanical response of the material, samples were deformed to steady state creep, unloaded to $10 \%$ of the original load, allowed to anneal for varying amounts of time, and reloaded to record the transients.

For TEM observations, the samples were cooled under load to preserve the dislocation structure produced by creep. Foils were cut from planes normal to the direction of stressing and were prepared by conventional jet polishing techniques. Stereo techniques were utilized to obtain a three dimensional view of the dislocation arrangements. With a double tilt holder stereo pairs were obtained by maintaining a constant diffraction condition while tilting an average of $10-20^{\circ}$. The average foil thickness in the vicinity of the observations was 1 to 1.5 times the precipitate diameter. In-situ annealing experiments were performed using a single tilt high temperature heating stage.

\section{Experimental Results}

Mechanical Response of CMSX-3 in the Creep Regime

A typical creep curve for $850^{\circ} \mathrm{C} / 552 \mathrm{MPa}$ is shown in Figure 1. A short primary transient is followed by steady state creep at a rate of $2.5 \times 10^{-8} / \mathrm{s}$. There is also a long period of accelerating tertiary creep, but this aspect of the creep problem will not be dealt with here.

Closer inspection of the response of the sample immediately after loading reveals an incubation period prior to the primary transient, as shown in Figure 2. At $850^{\circ} \mathrm{C} / 552$ $\mathrm{MPa}$ the incubation period is approximately 600 seconds in length. At $800^{\circ} \mathrm{C}$ the incubation is $1.6 \times 10^{4}$ seconds, while at $900^{\circ} \mathrm{C}$ it is immeasurably short.

In Figure 3 are the results of a recovery test at $850^{\circ} \mathrm{C} / 552 \mathrm{MPa}$. After steady state creep was reached, the load was reduced by $90 \%$ and the sample was annealed 


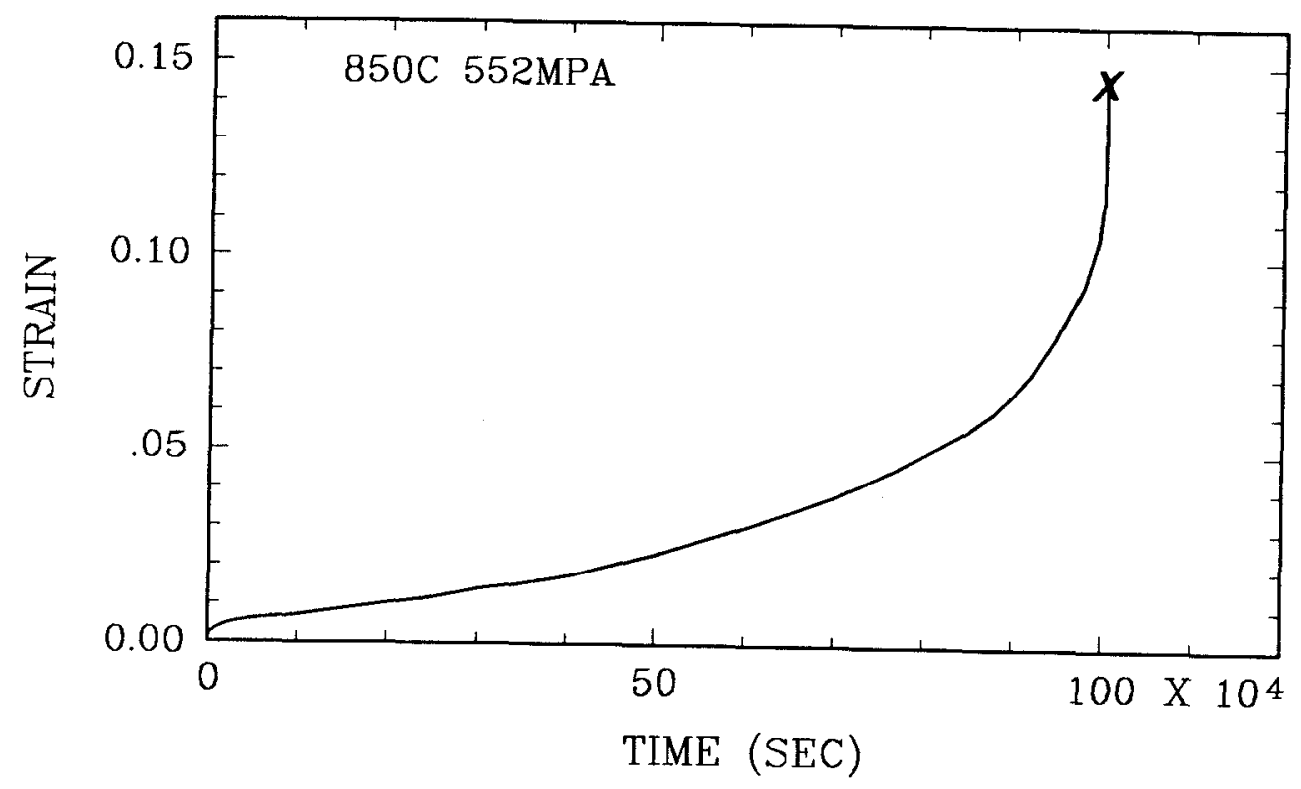

Figure 1 - Typical creep curve for $<001>$ oriented CMSX-3 at 850C/552MPa.

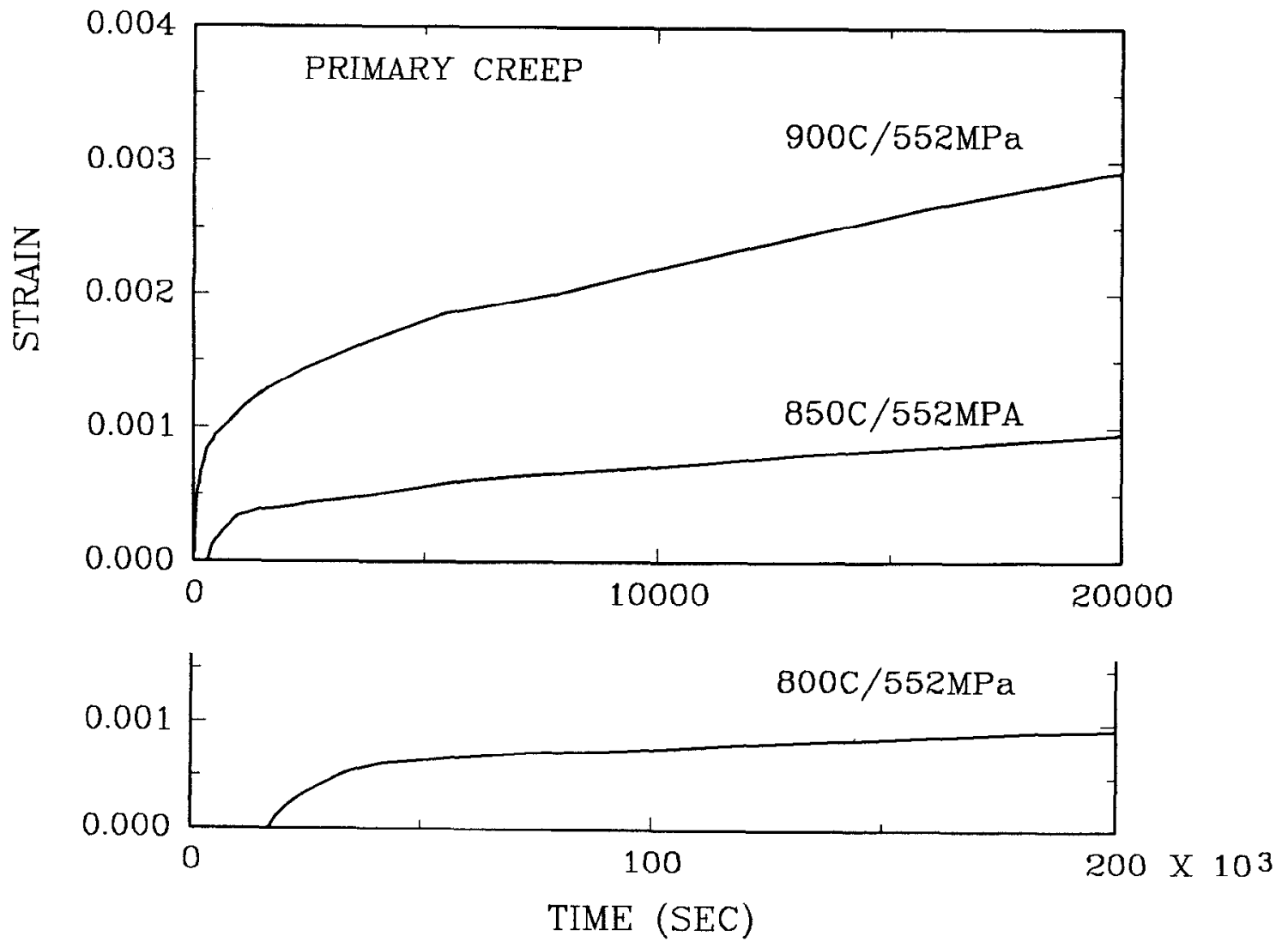

Figure 2 - Details of the primary transient. Note the incubation periods at 800 and $850^{\circ} \mathrm{C}$. 
for $1.62 \times 10^{4}$ seconds (4.5 hours). Upon reloading a very slight transient was observed, during which a strain of only $2.1 \times 10^{-4}$ was accumulated.

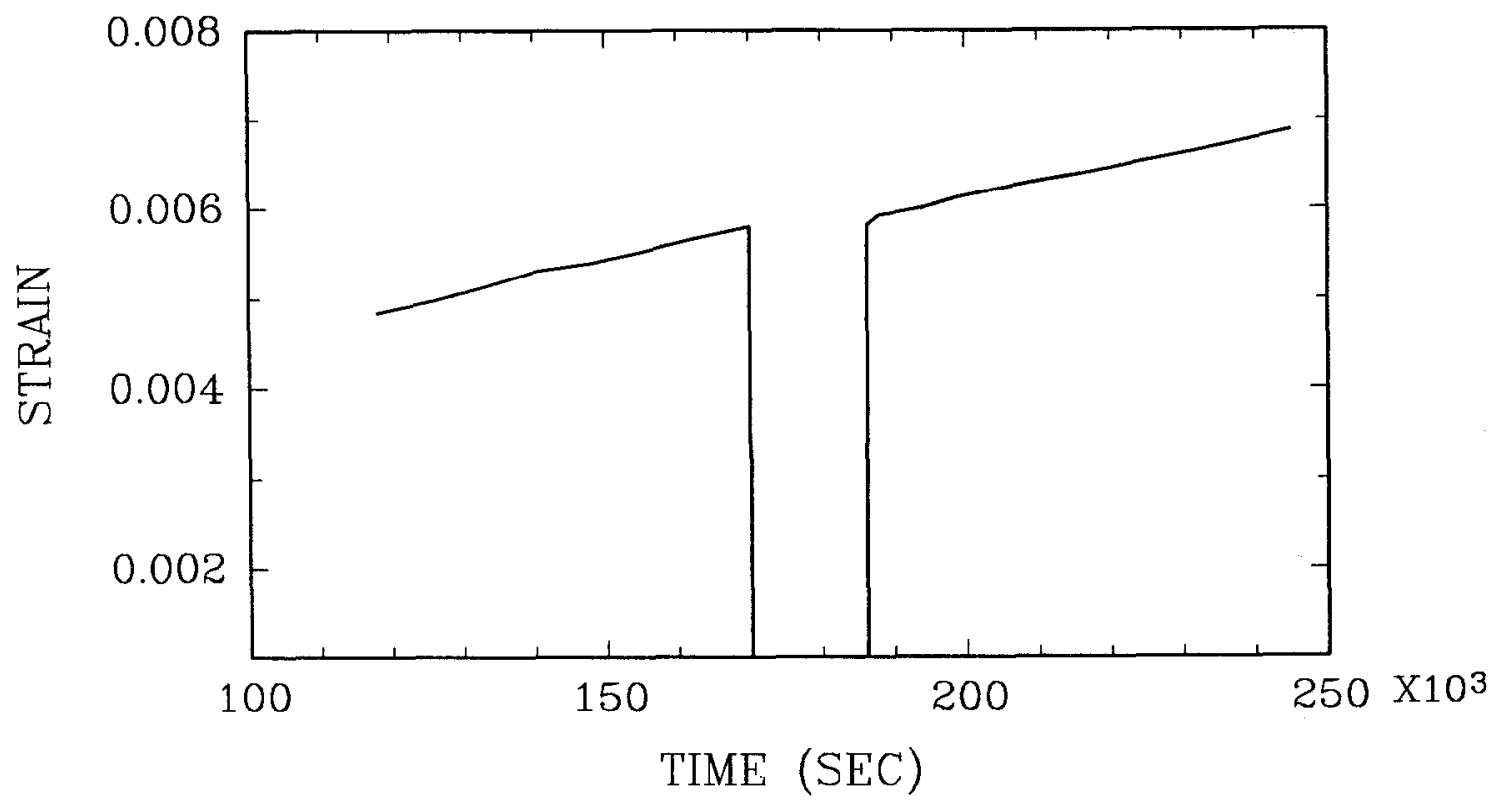

Figure 3 - The effect of static recovery on the reloading transient of a sample which was deformed to steady state creep, unloaded for $1.6 \times 10^{4}$ seconds, and then reloaded.

Dislocation Structures Produced by Creep and Recovery

In the aged condition, the single crystal material contains a very low density of dislocations. Over very large areas there are many precipitates which are completely coherent with the matrix with only an occasional dislocation found at the interface. In addition, there are occasional areas which show a tangled arrangement of dislocations that are associated with irregular shaped $\gamma^{\prime}$ particles. These areas of grown-in dislocations are widely spaced and at most one of these areas is encountered per TEM foil.

During primary creep the matrix material becomes filled with dislocations. Stereo pairs clearly show dislocations bowing between the $\gamma^{\prime}$ precipitates in the narrow matrix passages on $\{111\}$ planes. Figure 4 shows a typical primary creep structure after 2100 seconds at $850^{\circ} \mathrm{C} / 552 \mathrm{MPa}$. The precipitate labeled " $A$ " extends through the thickness of the foil and the dislocations in the matrix passages to the left and right are bowing out through the vertical matrix gaps, as shown schematically in Figure 5(a). In other areas such as the ones marked " $B$ " and " $C$ ", a matrix gap has been completely captured in the plane of the foil and the dislocations are bowing into the horizontal gaps, as schematically illustrated in Figure 5(b). Early in the primary transient all of the dislocations in any local field of view are found to have the same Burgers vector, and a single dislocation line can be traced over the distance of many $\gamma^{\prime}$ particles. As the end of the primary transient is approached dislocations with different Burgers vectors from neighboring regions penetrate locally and react with dislocations of the primary system; see Figures 6(a) and (b) where the same area is imaged for two different diffraction conditions, displaying two different sets of dislocations.

The steady state dislocation structure is characterized by a complicated three dimensional nodal network, pictured in Figure 7(a). The network contains at least three different sets of dislocations. Again this dense dislocation network is completely contained in the matrix, and there are very few dislocations in the matrix passages which 
are not associated with the network. The gamma prime precipitates are occasionally observed to be sheared by pairs of dislocations in the later stages of steady state creep.

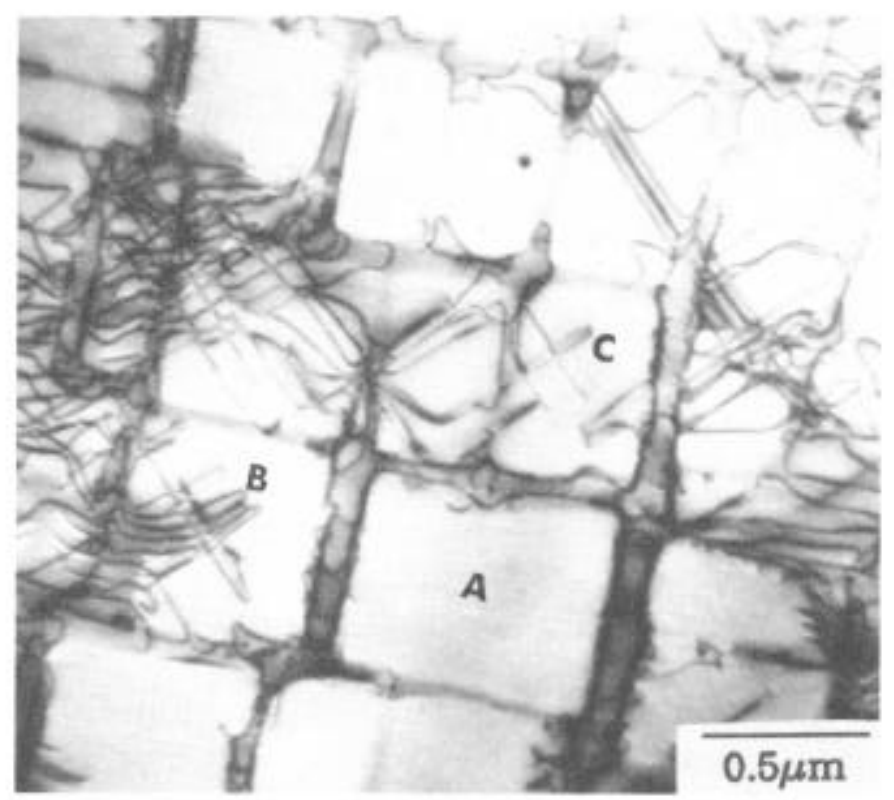

Figure 4 - Dislocation structure during primary creep. Dislocations in the matrix on either side of " $A$ " are bowing through vertical gaps, while near " $B$ " and " $C$ " a horizontal matrix gap with dislocations is contained in the plane of the foil.
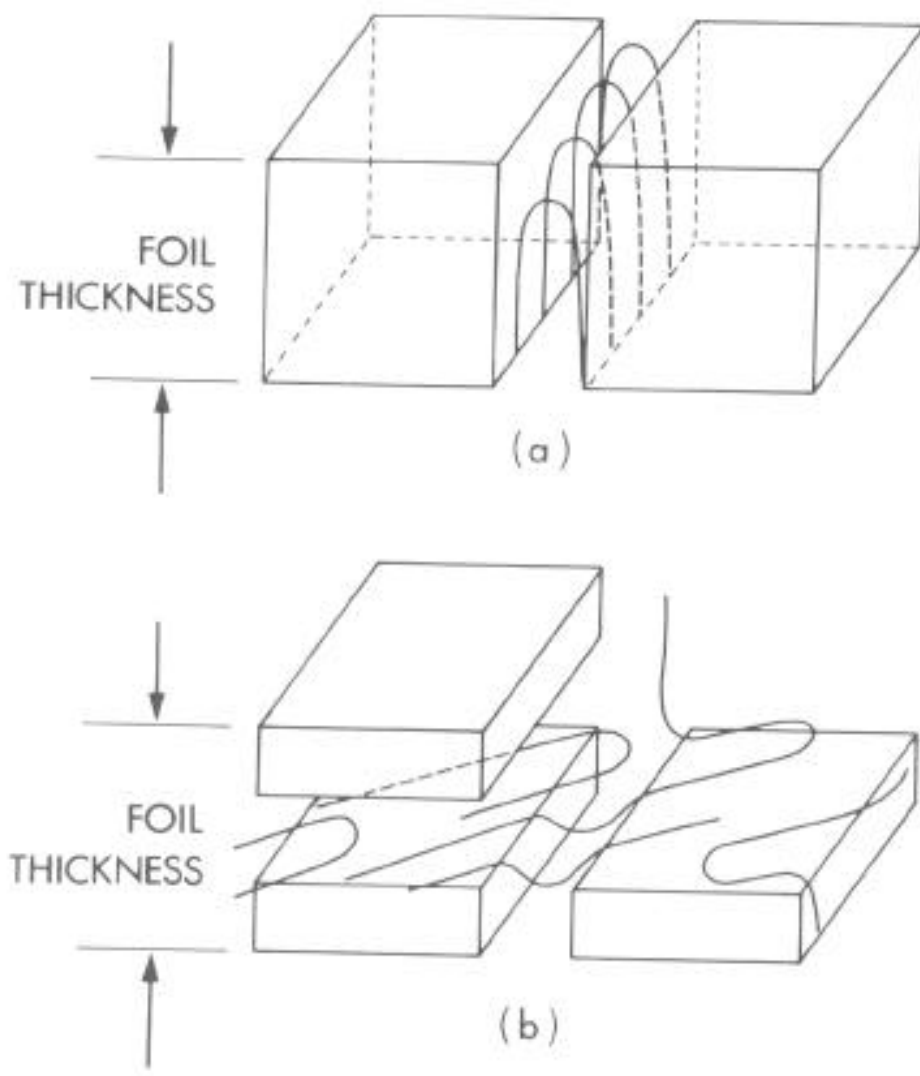

Figure 5 - (a) Schematic 3-D view of dislocations bowing through vertical gaps and (b) a horizontal gap contained in the foil with dislocations bowing through the gap captured in the plane of the foil. 
After creeping to steady state a sample was bulk annealed under no stress for 750 hours. From Figure $7(\mathrm{~b})$, it is obvious that the overall density of dislocations has decreased and the network has coarsened. Although the network appears to be more planar, it is not necessarily at the $\gamma / \gamma^{\prime}$ interface. In-situ static annealing at $850^{\circ} \mathrm{C}$ and $900^{\circ} \mathrm{C}$ with a heating stage also shows a gradual decrease in the local dislocation density associated with the three dimensional network at $850^{\circ} \mathrm{C}$ for annealing times of 2 to 3 hours, but the kinetics of this dislocation annihilation process at these short times at $850^{\circ} \mathrm{C}$ do not necessarily suggest that static recovery is fully responsible for this short time clearing up of the structure. However, at $900^{\circ} \mathrm{C}$ the rate of decrease of dislocation density does suggest that some diffusion controlled static recovery is operative. It is necessary to be careful in interpreting the results obtained with thin foils, since dislocations are able to escape from the free surface. A more thorough analysis of the in-situ static recovery processes and the problem of surface effects in thin films will be presented in the future.

\section{Finite Element Model of the Two Phase Microstructure}

A finite element model of the $\gamma / \gamma^{\prime}$ microstructure was constructed to observe the changing distribution of stress, strain and pressure in the structure due to the creep process. The model used generalized plane strain elements, which permit the development of a uniform strain in the third dimension. The symmetry of the problem allows one quarter of the precipitate plus matrix to be modeled. The top and right boundaries are constrained to remain plane to satisfy overall symmetry in the microstructure. The finite element mesh is shown in Figure 8(a). Misfit was incorporated into the model by assigning to the $\gamma$ and $\gamma^{\prime}$ their respective coefficients of thermal expansion. The coefficients were estimated using the data of Grose and Ansell (2) and Giamei et al. (3). At $850^{\circ}$ the misfit is $-3.05 \times 10^{-3}$, which is slightly lower than the value reported by Fredholm and Strudel (4), where they measured dislocation spacings after aging of CMSX-2 at $1050^{\circ} \mathrm{C}$. Anisotropic elastic constants for the $\gamma$ and $\gamma^{\prime}$ were also used in the analysis. Since the TEM stereo observations showed all the deformation to be occurring in the matrix material, the $\gamma^{\prime}$ precipitate is constrained to remain elastic. The finite element program utilizes a simple power law creep relation in its calculations. The analysis was completed for the separate cases of assigning to the matrix the macroscopic creep properties of the superalloy, and for the case of assigning to the matrix the creep properties of a $\mathrm{Ni}-\mathrm{W}$ solid solution (6).

Since the finite element analysis provides a continuum solution to the two phase material deformation problem, it does not provide much insight to the the primary creep process, which is clearly a local dislocation mechanics problem of filling the initially undeformed material with dislocations by bowing them out between precipitates on selected slip systems. However, with the buildup of the uniformly dense dislocation networks in the matrix as a result of slip on multiple systems, the finite element analysis can provide useful insight to the source of the steady state creep resistance that comes from the constraint of the non-deforming $\boldsymbol{\gamma}^{\prime}$ precipitates.

As a result of the misfit, the matrix material is initially in hydrostatic compression, while the precipitate is in hydrostatic tension. The equivalent misfit stresses in the matrix are on average around $455 \mathrm{MPa}$. Upon loading the material $\left(850^{\circ} \mathrm{C} / 552 \mathrm{MPa}\right.$ ) there is a rapid transient, during which the misfit stresses are relaxed. As the creep proceeds, there is a relaxation of the matrix equivalent stresses (equal to the resolved shear stresses on $\{111\}<110>$ systems) with the buildup of a pressure gradient in the matrix. In the vertical channels (parallel to the applied stress) there is a positive pressure buildup, while in the horizontal channels (normal to the applied stress) a negative pressure builds up from the center of the face of the precipitate, providing load support. Decreasing the matrix creep resistance leads to shorter relaxation times and an increased macroscopic creep rate which develops at long times $t / \tau>5$. (The macroscopic creep rate is calculated from the displacements of the upper boundary of the mesh with respect to the lower, and $\tau$ is a relaxation time equal to the time to 


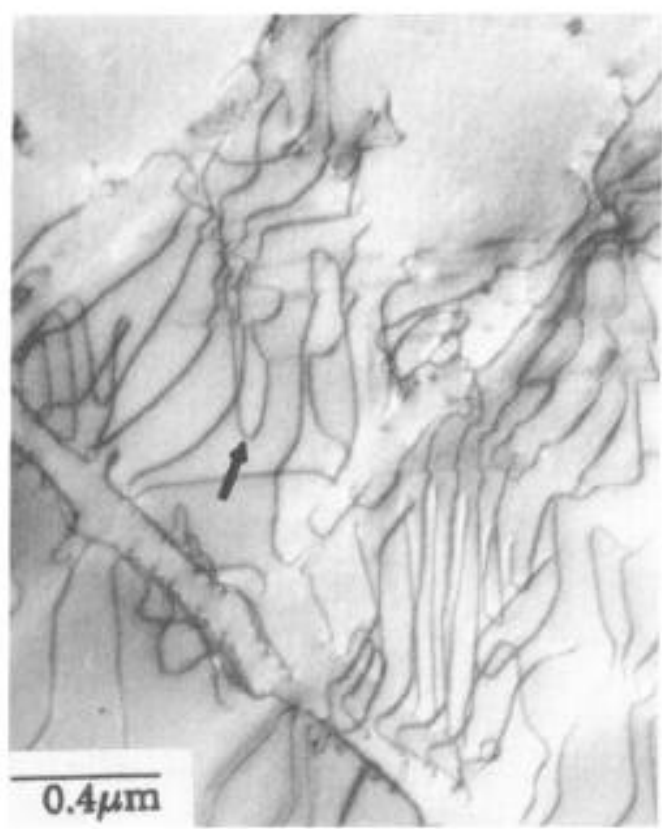

(a)

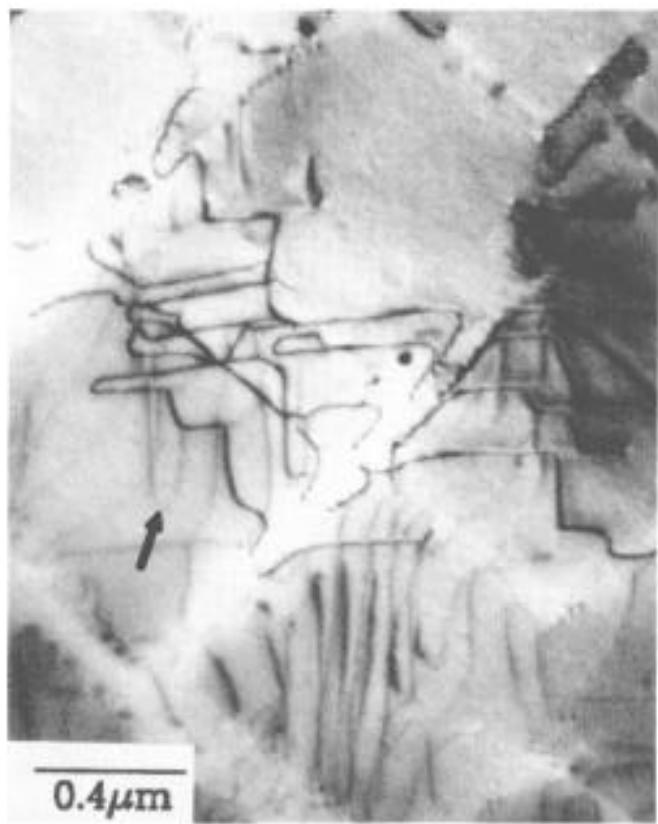

(b)

Figure 6 - Dislocations in (b) with a Burgers vector different from the primary dislocations in (a) begin to interpenetrate and form nodes. The two sets of dislocations are imaged for (a) $\mathbf{g}=\overline{2} 00$ and (b) $\mathrm{g}=020$. The arrows mark a common reference point in each micrograph.

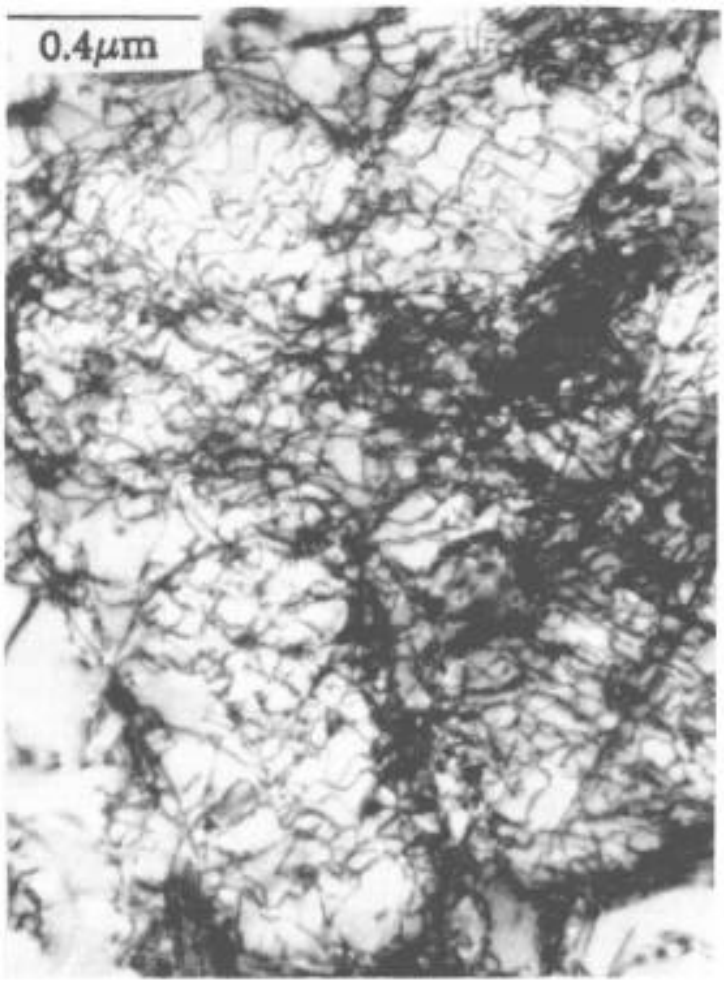

(a)

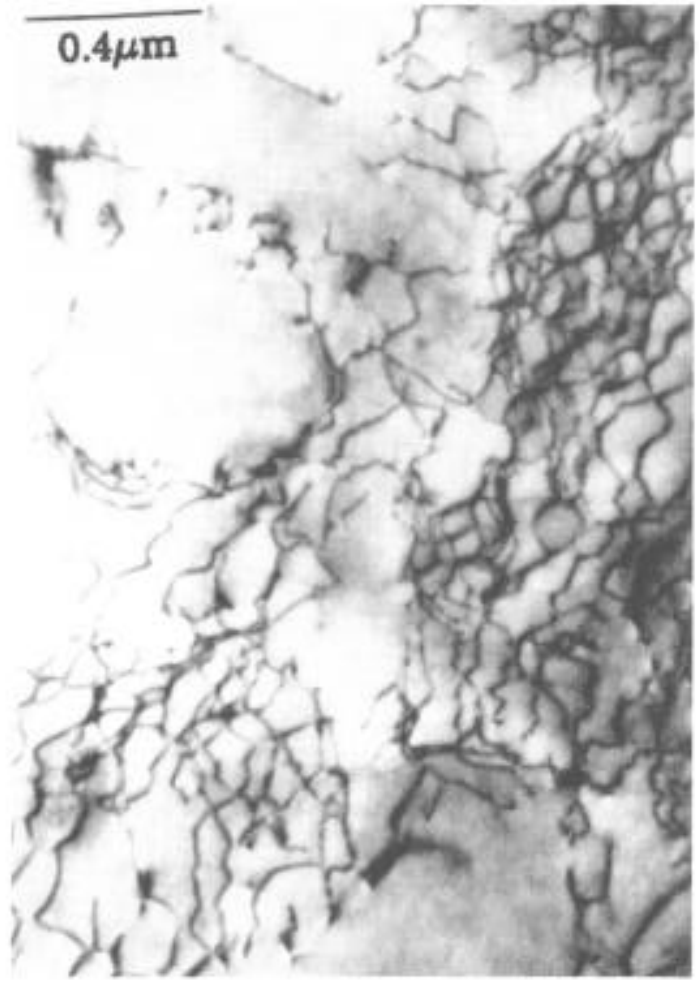

(b)

Figure 7 - (a) Characteristic steady state dislocation structure with a dense three dimensional nodal network in the matrix. (b) Coarsening of the three dimensiona' network following 750 hours of bulk static annealing. The scale in these two micrographs is slightly different. 
accumulate creep strains of the order of the elastic strains.)

As the creep continues in the matrix, the equivalent stresses in the elastic gamma prime precipitate continue to rise. Contours of stresses resolved onto $\{111\}<110>$ slip systems in the precipitate are shown in Figure $8(b)$ for $t / \tau=485$. As noted on the contour plot, the maximum resolved shear stress is $281 \mathrm{MPa}$ which when divided by the Schmid factor gives a tensile stress of $689 \mathrm{MPa}$. The maximum stresses are just below the corner of the cube, approximately $1 / 4$ of the distance along the cube edge. It is interesting to note that late in steady state creep the precipitates are sometimes observed to be sheared by dislocations entering at this very location, as shown in Figure 9.

\section{Discussion}

For a given creep rate, a single crystal nickel base alloy with a high volume fraction of $\gamma^{\prime}$ has a tensile creep resistance which is an order of magnitude higher than the creep resistance of either the $\gamma$ or $\gamma^{\prime}$ materials in bulk. For example, for a creep rate of $2.5 \times 10^{-8} / \mathrm{s}$ the creep resistance of $\mathrm{Ni}_{3} \mathrm{Al}$ is only about $60-70 \mathrm{MPa}(5)$, and the resistance of a $\mathrm{Ni}-\mathrm{W}$ solid solution is on the order of $30-40 \mathrm{MPa}(6)$, while the creep resistance of CMSX-3 is $550 \mathrm{MPa}$. The reasons for this order of magnitude improvement in creep resistance of the two phase alloy become more clear when the development of the steady state structure is followed by TEM. Stereo pairs show that all deformation is occurring in the matrix. Apparently at the stress levels studied here or at lower stresses, the difficulty of pushing a pair of normal $\gamma$ phase dislocations into the ordered gamma prime phase is so high that the dislocations are confined to the matrix. Since the matrix in the aged condition contains so few dislocations, the development of the plastic response requires generation of dislocations from limited sources and spreading by bowing them out between the precipitates. For this reason, an incubation period may be required and large areas of dislocations of a single Burgers vector are seen in the early stages of primary creep. For a matrix passage with a thickness of $60 \mathrm{~nm}$ and deformation on a $\{111\}<110>$ system, the Orowan stress is $167 \mathrm{MPa}$, which gives a tensile deformation resistance of $408 \mathrm{MPa}$. This is clearly the major contribution to the deformation resistance of the two phase alloy. The quantitative aspects of the kinematics of the spreading of the dislocations and the resistance of the gamma prime to shearing by precipitates have not yet been treated, but will be necessary for the development of a model for the constitutive creep response of the material.

As the dislocations continue to fill the material during the primary transient, dislocations spreading from widely spaced sources in neighboring regions begin to interpenetrate and react, thereby preventing large crystallographic rotations. Eventually a dense three dimensional nodal network, characteristic of steady state creep, is built up in the matrix. At temperatures around $850^{\circ} \mathrm{C}$ annealing shows the static recovery process to be sluggish, producing only very slight reloading transients. At this temperature and at lower temperatures, a combination of static and dynamic recovery appears to be required for the maintenance of the steady state creep process. At temperatures around $900^{\circ} \mathrm{C}$ and higher the steady state creep process is probably controlled by static recovery.

As the steady state creep process proceeds, the constraint of the non-deforming precipitates leads to the buildup of a negative pressure in the matrix passages normal to the applied stress. This provides additional load support for the creeping matrix, leading to a much reduced creep rate relative to the unconstrained creep behavior of the solid solution material. This effect can be compared to the more familiar problem of compressing a thin disk of material between two rigid dies; where the flow constraint due to wall friction produces an increased load carrying ability of the disk due to pressure buildup. This problem has been discussed in an earlier paper (7).

In the later stages of steady state creep, as the stresses in the matrix relax and the stresses in the non-deforming $\gamma^{\prime}$ continue to rise, the precipitates may be sheared by 


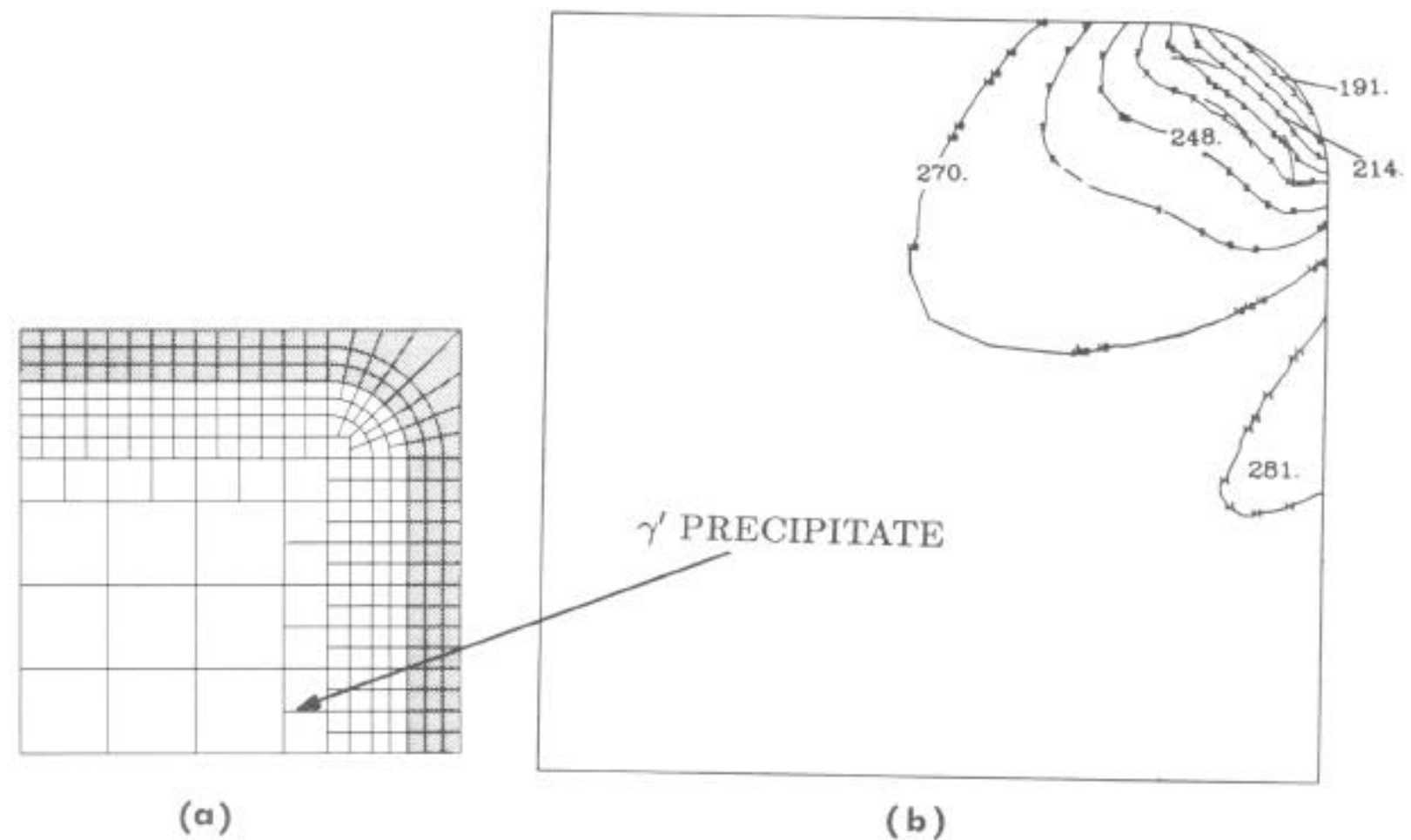

Figure 8 - The $\gamma / \gamma^{\prime}$ finite element mesh (a), with the shaded region corresponding to the matrix and the unshaded to the precipitate. The resolved shear stresses on $\{111\}<110>$ systems in the precipitate are shown in (b).

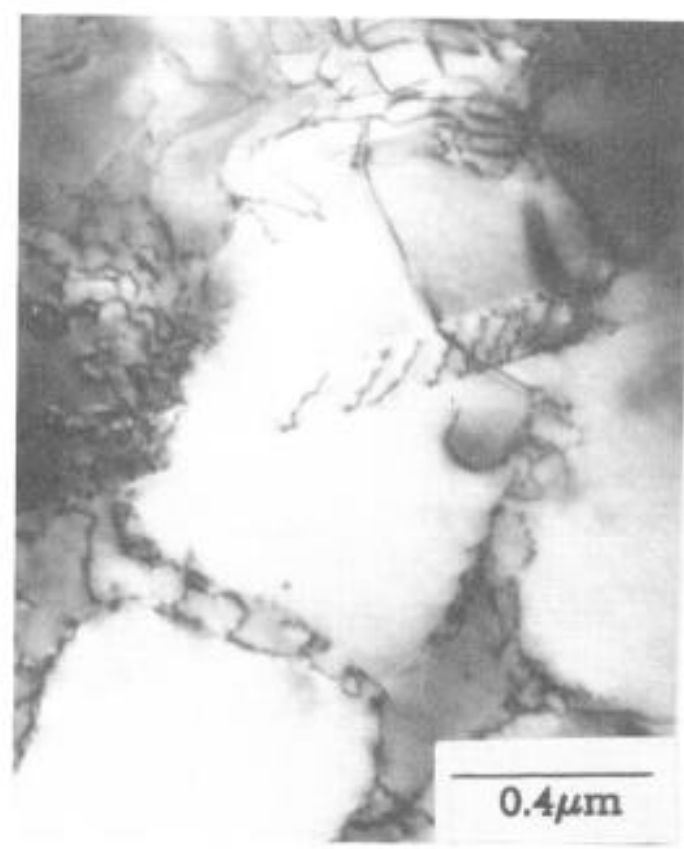

Figure 9 - Dislocations shearing the $\gamma^{\prime}$ precipitate in the later stages of steady state creep. Note that the point of entry corresponds to the point of maximum resolved shear stresses in Figure 8. 
dislocations. For the conditions studied here this was only an occasional event, but for higher stresses it is expected that this would be observed more frequently.

These results suggest that for alloys with a high volume fraction of $\gamma^{\prime}$ (at a constant volume fraction), non-shearable cuboidal precipitates which are spaced as closely as possible will provide optimum creep resistance. This was shown to be true for CMSX-2 by Caron and Khan (8) and Khan (9) where irregular shaped precipitates are sheared by dislocations, giving a lower creep resistance (8), and also where larger cuboidal precipitates (and therefore larger matrix passages) produce a lower creep resistance (9). This in turn implies that any compositional adjustments which influence the level of misfit between the two phases, the composition of the $\gamma^{\prime}$, the equilibrium volume fraction of $\gamma^{\prime}$, or its coarsening behavior, will have major resultant influences on the creep properties, by changing the $\gamma^{\prime}$ morphology or shape, or the matrix gap dimension. Compositional changes will also affect the solid solution matrix creep properties, which in turn influence the overall creep properties, but to a lesser extent than the above mentioned factors.

\section{Acknowledgements}

This research was supported by the NSF/MRL under Grant No. DMR 84-18718, through the Center for Materials Science and Engineering at M.I.T. We are grateful to Dr. Mehmet Doner of the Allison Division of the General Motors Corporation for supplying the single crystals.

\section{$\underline{\text { References }}$}

1. K.L. Harris, G.L. Erickson, and R.E. Schwer in Superalloys 1984 (Warrendale, PA : The Metallurgical Society, 1984), 227, M. Gell, et al., eds.

2. D.A. Grose and G.S. Ansell, Met. Trans. 12A, 1981, 1631.

3. A.F. Giamei, D.D. Pearson, D.L. Anton in High Temperature Ordered Intermetallic Alloys, MRS Symp. Proc. Vol. 39, (Pittsburg, PA: Materials Research Society, 1986), 293, C.C. Koch et al., eds.

4. A. Fredholm and J.L. Strudel in Superalloys 1984, (Warrendale, PA :The Metallurgical Society, 1984), 211, M. Gell, et al., eds.

5. D.M. Shah, Scripta Met. 17, $1983,997$.

6. W.R. Johnson, C.R. Barrett and W.D. Nix, Met. Trans. 3A, 1972, 963.

7. A.S. Argon, A.K. Bhattacharya, T.M. Pollock in Constitutive Relations and Their Physical Basis, Proc 8th RISO Intl. Symp., (Roskilde, Denmark: Riso National Laboratory, 1987), 39, .S.I. Anderson, et al., eds.

8. P. Caron and T. Khan, Materials Sci. and Engr. 61, 1983, 173.

9. T. Khan in High Temperature Alloys for Gas Turbines and Other Applications 1986, (Dordrecht, Holland: D. Riedel Publishing), 21, W. Betz, et al., eds. 